\title{
Chemical spots and their dynamical evolution on HgMn stars
}

\section{Heidi Korhonen ${ }^{1}$, Swetlana Hubrig ${ }^{2}$, Maryline Briquet $^{3}$, Federico González ${ }^{4}$, and Igor Savanov ${ }^{5}$}

\author{
${ }^{1}$ European Southern Observatory, Karl-Schwarzschild-Str 2, D-85748, Garching bei München, \\ Germany \\ email: hkorhone@eso.org \\ ${ }^{2}$ Astrophysikalisches Institut Potsdam, An der Sternwarte 16, D-14482 Potsdam, Germany \\ ${ }^{3}$ Instituut voor Sterrenkunde, Katholieke Universiteit Leuven, Celestijnenlaan 200 D, \\ B-3001 Leuven, Belgium \\ ${ }^{4}$ Instituto de Ciencias Astronomicas, de la Tierra, y del Espacio (ICATE), \\ 5400 San Juan, Argentina \\ ${ }^{5}$ Institute of Astronomy, Russian Academy of Sciences, Pyatnitskaya 48, \\ Moscow 119017, Russia
}

\begin{abstract}
Our recent studies of late B-type stars with HgMn peculiarity revealed for the first time the presence of fast dynamical evolution of chemical spots on their surfaces. These observations suggest a hitherto unknown physical process operating in the stars with radiative outer envelopes. Furthermore, we have also discovered existence of magnetic fields on these stars that have up to now been thought to be non-magnetic. Here we will discuss the dynamical spot evolution on HD 11753 and our new results on magnetic fields on AR Aur.
\end{abstract}

Keywords. Stars: atmospheres, chemically peculiar, early-type, magnetic fields, spots

\section{Introduction}

Recently, Kochukhov et al. (2007), using Doppler Imaging technique, reported a discovery of secular evolution of the mercury distribution on the surface of the HgMn star $\alpha$ And. However, this result was never verified by other studies due to the lack of observational data. Until very recently, the only other $\mathrm{HgMn}$ star with a published surface elemental distribution was AR Aur (Hubrig et al. 2006), where the discovered surface chemical inhomogeneities are related to the relative position of the companion star. The elements $\mathrm{Y}$ and $\mathrm{Sr}$ are strongly concentrated in an equatorial ring, which has a gap exactly on the area permanently facing the secondary.

A large number of spectra of a sample of $\mathrm{HgMn}$ stars were obtained with the CORALIE spectrograph at the $1.2 \mathrm{~m}$ Euler telescope on La Silla during a programme dedicated to a search for SPB-like pulsations in B-type stars. In Briquet et al. (2010) we published two sets of surface maps of HD 11753 based on these data. The maps are separated by approximately two months and are obtained from three different elements: Y II, Ti II and Sr II. The maps made from the Y II $4900 \AA$ line (see Fig. 1) exhibit a high abundance region at phases $0.5-1.0$ extending from the latitude $45^{\circ}$ to the pole, with an extension to the equator around the phase 0.8 . The Y II abundance distribution shows also a high latitude lower abundance spot around phases $0.2-0.4$. Clear evolution in the surface features is present during the two months that separate the datasets. The lower abundance high latitude feature at phases $0.2-0.4$ becomes more extended and less prominent in the 

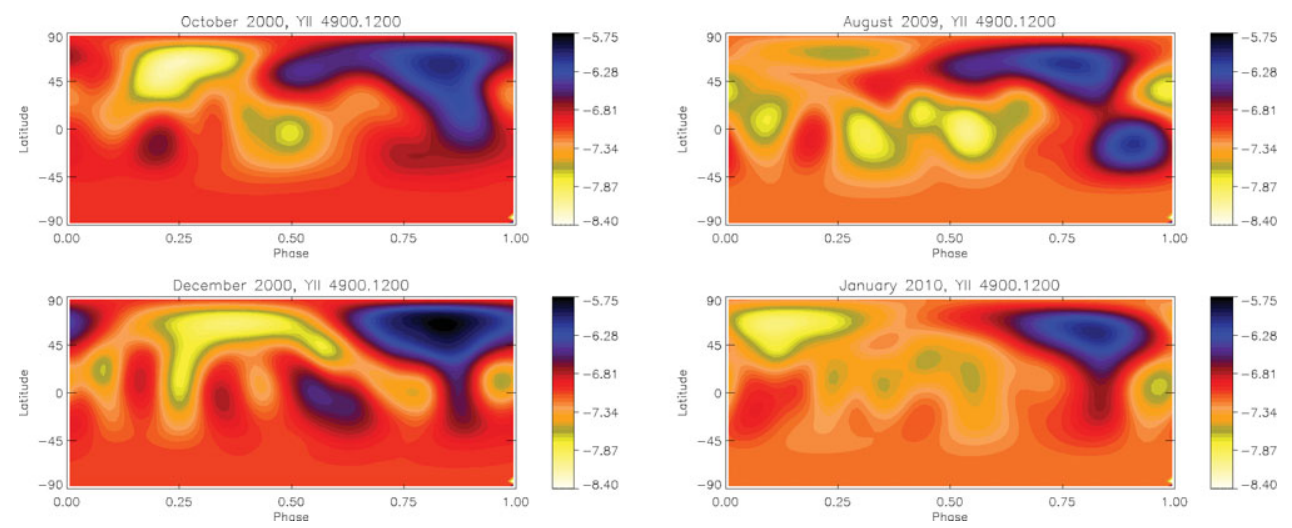

Figure 1. Chemical surface maps of HD 11753 from the Y II $4900 \AA$ A line at four different epochs. In the maps the abscissa is the longitude in phases and the ordinate is the latitude in degrees. Colour indicates different abundances, with darker denoting higher abundance.

second set, while the abundance of the high abundance spot at phases $0.6-1.0$ gets more prominent with time.

We have also obtained measurements of the magnetic field strength with the moment technique using several elements in a circularly polarised high resolution spectrum of another HgMn star, AR Aur. These observations revealed the presence of a longitudinal magnetic field in both stellar components (Hubrig et al. 2010).

Here, we continue the investigation of HD 11753 using newer CORALIE data from 2009 and 2010, and discuss the magnetic field measurements of AR Aur.

\section{Spot evolution in HD11753 for 2000-2010}

HD 11753 is a single-lined spectroscopic binary with an effective temperature of 10612 K (Dolk et al. 2003). According to our observations the orbital period of the binary would be long, and the projected rotational velocity vsin $i=13.5 \mathrm{~km} / \mathrm{s}$ (Briquet et al. 2010). After adding the new 2009 and 2010 datasets the rotational period is improved to $\mathrm{P}=9.531 \mathrm{~d}$ (Korhonen et al. 2010).

We have obtained Doppler images of HD 11753 from CORALIE spectra for four different epochs. In Doppler imaging spectroscopic observations at different rotational phases are used to measure the rotationally modulated distortions in the line-profiles. These distortions are produced by the inhomogeneous distribution of a surface characteristic, e.g., surface temperature or element abundance. Surface maps are constructed by combining all the observations from different phases and comparing them with synthetic model lineprofiles. For accurate Doppler imaging the shape and changes of the line-profile have to be well defined. This requires high resolving power and high signal-to-noise-ratio.

The Doppler images of HD 11753 using the Y II $4900 \AA$ line are shown in Fig. 1. The two first maps, both from 2000, have 65 days in between them. Clear temporal evolution of the chemical spots occurs even on such short timescales. The high abundance spot around the phase 0.75 gets more concentrated with time, and the lower abundance spot around the phase 0.25 more extended. These two maps were already published by Briquet et al. (2010), but the August 2009 and January 2010 maps are previously unpublished. These latter maps have approximately four and half months in between them, and again they show temporal evolution of the surface structures. The high abundance spot of phase 0.75 is at high latitudes much more extended in August 2009 than in January 
2010. Also, the equatorial high abundance spot seen at phase 0.85 in the August 2009 map has disappeared before January 2010. The lower abundance spot of phase 0.0-0.4 is almost non-existent in August 2009, but clearly present in January 2010. However, the August 2009 dataset has a large phase gap, 0.17-0.47, close to the phase of the lower abundance spot. Our tests show, though, that a phase gap of 0.3 in phase does not affect the recovery of such large surface features (see Korhonen et al. (2010)).

All in all, the chemical spots retain their position on the stellar surface stably the almost 10 year period our observations cover. The exact shape changes, though, and this change happens even on time scales of months.

\section{Magnetic field in AR Aur}

The double-lined spectroscopic binary AR Aur has an orbital period of $4.13 \mathrm{~d}$. It is a young system with an age of only $4 \times 10^{6} \mathrm{yr}$ and its primary, showing HgMn peculiarity, is exactly on the Zero Age Main Sequence while the secondary is still contracting towards it. Variability of spectral lines associated with a large number of chemical elements was reported for the first time for the primary component of this eclipsing binary by Hubrig et al. (2006).

Doppler maps for the elements $\mathrm{Mn}, \mathrm{Sr}, \mathrm{Y}$, and $\mathrm{Hg}$ using nine spectra of AR Aur observed at the European Southern Observatory with the UVES spectrograph at UT2 in 2005 were for the first time presented at the IAU Symposium 259 by Savanov et al. (2009). To prove the presence of a dynamical evolution of spots also on the surface of AR Aur, we obtained new spectroscopic data with the Coudé Spectrograph of the $2.0 \mathrm{~m}$ telescope of the Thüringer Landessternwarte and the SES spectrograph of the $1.2 \mathrm{~m}$ STELLA-I robotic telescope at the Teide Observatory. A number of SOFIN spectra of AR Aur were obtained in 2002 at the Nordic Optical Telescope, which we also used in our analysis. Our new results show secular evolution of the chemical spots on AR Aur (Hubrig et al. 2010). Fig. 2 shows Sr II 4215.5 $\AA$ line during three different epochs (late 2002 , late 2005 , and late 2008 - early 2009 ), but at the same rotational phase, $\sim 0.8$. The shapes of the lineprofiles are clearly different indicating that also the Sr spots very likely changed their shape and abundance with time.

To pinpoint the mechanism responsible for the surface structure formation in HgMn stars, we carried out spectropolarimetric observations of AR Aur and investigated the presence of a magnetic field during a rotational phase of very good visibility of the spots of overabundant elements. The spectropolarimetric observations of AR Aur at the rotation phase 0.622 were obtained with the low-resolution camera of SOFIN $(R \approx 30000)$ at the Nordic Optical Telescope. Since most elements are expected to be inhomogeneously distributed over the surface of the primary of AR Aur, magnetic field measurements were carried out for samples of Ti, Cr, Fe, and Y lines separately. Among the elements showing line variability, the selected elements have numerous transitions in the observed optical spectral region, allowing us to sort out the best samples of clean unblended spectral lines with different Landé factors.

Our magnetic field measurements, which are discussed in detail by Hubrig et al. (2010), were done using the formalism described by Mathys (1994). A longitudinal magnetic field at a level higher than $3 \sigma$ of the order of a few hundred Gauss is detected in Fe II, Ti II, and Y II lines, while a quadratic magnetic field $\langle B\rangle=8284 \pm 1501 \mathrm{G}$ at $5.5 \sigma$ level was measured in Ti II lines. No crossover at $3 \sigma$ confidence level was detected for the elements studied. Further, we detect a weak longitudinal magnetic field, $\left\langle B_{z}\right\rangle=-229 \pm 56 \mathrm{G}$, in the secondary component using a sample of nine Fe II lines. The main limitation on the accuracy achieved in our determinations is set by the small number of lines that can 


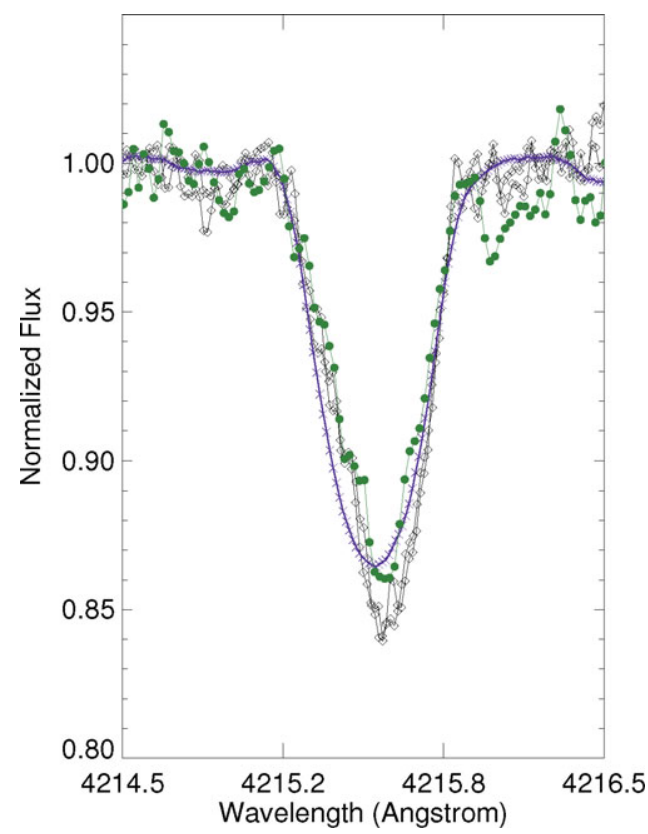

Figure 2. Line profiles of the Sr II $4215.5 \AA$ line at the rotation phase $\sim 0.8$ for late 2002 (open diamonds), late 2005 (crosses), and late 2009 - early 2010 (filled circles) show clearly different shapes.

be used for magnetic field measurements. The diagnosis of the quadratic field is more difficult than that of the longitudinal magnetic field, and it depends much more critically on the number of lines that can be used for the analysis.

\section{Summary}

The fast dynamic evolution of the spots on HD 11753 implies hitherto unknown physical mechanism operating in the outer envelopes of late B-type stars with HgMn peculiarity and the detection of the magnetic field in AR Aur shatters the traditional view that HgMn stars do not exhibit magnetic fields. For the proper understanding of the nature of these stars we need accurate information on the element spot configuration and underlying magnetic fields in a sample of HgMn stars to determine a link between these properties and stellar fundamental parameters such as rotation rate, temperature, evolutionary state, stellar mass, multiplicity and orbital parameters. It is clear that time series of high resolution spectropolarimetric observations are needed to solve the puzzle these stars represent.

\section{References}

Briquet, M., Korhonen, H., González, J. F., Hubrig, S., \& Hackman, T. 2010, A\&3A, 511, A71 Dolk, L., Wahlgren, G. M., \& Hubrig, S. 2003, A\& $A$ 402, 299

Hubrig, S., González, J. F., \& Savanov, I., et al. 2006, MNRAS, 371, 1953

Hubrig, S., Savanov I. \& Ilyin, I., et al. 2010, MNRAS, 408, L61

Kochukhov, O., Adelman, S. J., Gulliver, A. F., \& Piskunov, N. 2007, Nature Physics, 3, 526

Korhonen, H., González, J. F., \& Briquet, M., et al. 2010, A\&A A, in prep.

Mathys G. 1994, A\&AS, 108, 547

Savanov I. S., Hubrig S., González J. F., Schöller M. 2009, IAU Symp. 259, p. 401 


\section{Discussion}

Morin: Have you tried to fit both datasets with a unique Doppler map to assess variability?

Korhonen: Oh, no, that's actually a good idea to try it. Thanks.

OLEG: Have you tried to use more than one spectral line for a construction of abundance maps?

Korhonen: Yes, I have. But then - but I also, as you well know, I mean there's also radial stratification of the atmosphere. So you have to be careful which lines you choose to.

Q.: Well, actually, not in these stars. But what I meant was is, if you take one individual lines and reconstruct an abundance map and take another interim line and reconstruct another map from the same data set and then compare the two maps, that would be another way to see if - if you have significant effects related to specific lines?

Korhonen: I agree. The problem is that it's difficult to find lines that are unblended; but yes, we are also working on that. 